\section{Presidential address 2017; Stepping in to the future}

\section{Dr Darshani Wijayawickrama \\ https://orcid.org/0000-0002-5920-4919}

Presidential address at the inaugural session of the Sri Lanka College of Sexual Health and HIV Medicine (Sri Lanka CoSHH), 5th, October 2017, Hilton, Colombo.

Chief Guest Professor Harendra de Silva, Guest of honour Dr Philip Hay, Distinguished foreign and local guests, Past presidents, council members and the members of the Sri Lanka College of Sexual health and HIV Medicine, My teachers, colleagues, ladies and gentlemen,

I am honoured to stand here today as the president of the Sri Lanka College of Sexual Health and HIV Medicine. Indeed, more than honoured, I am humbled.

The theme of the $22^{\text {nd }}$ annual scientific sessions and of my speech is 'Stepping in to the future'. I would like to step swiftly across the mile stones of the past, the present and towards future.

It was June, 1981 when the Centres for Disease Control and Prevention published its first report about the mysterious deaths of five young, previously healthy gay men. Since then, More than three and a half decades have elapsed.

Nearly 37 million of people were living with HIV by the end of 2016

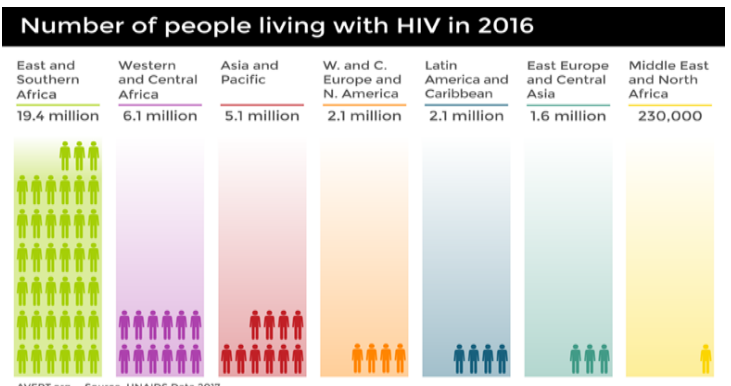

In 2016, there were roughly 1.8 million new HIV infections. That was a decline from 2.1 million new infections in 2015.

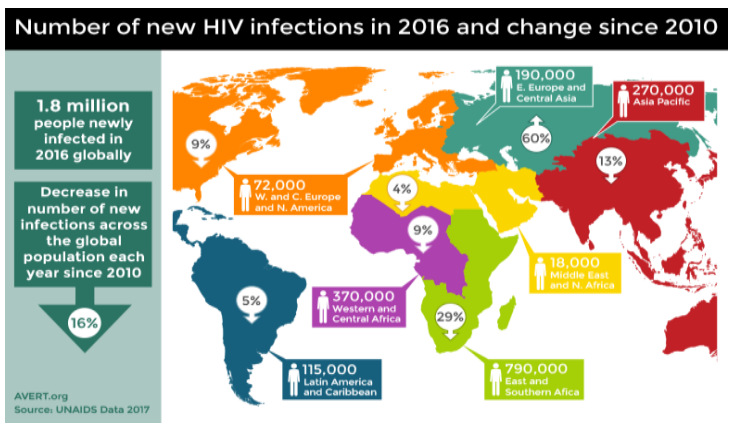

A slightly more positive trend has emerged, as new infections among adults are estimated to have declined by 2 fold - i.e. the decline between 2010 and 2016 has been 16\% for the general population, whereas there was only an $8 \%$ decline between 2010 and 2015. New infections among children have halved from 300,000 in 2010 to 160,000 in 2016 (47\%).

Despite the progress made across the 69 countries which witnessed a decline of $50 \%$ or more in new HIV infections over the last 10 years, many have made no measurable progress. Yet some countries are still experiencing worrying increases in new HIV infections.

Since 2010, the annual number of new infections in the Eastern Europe and Central Asia region, for example, has climbed by an alarming $60 \%$.

The two key concepts that dominated the public health aspect regarding HIV during the 2010s were biomedical prevention and HIV care continuum.

Bio medical prevention refers to the use of antiretrovirals (ARVs) to prevent transmission, either as treatment as prevention (TasP), whereby HIV is fully suppressed, rendering it virtually impossible to transmit, or as preexposure prophylaxis (PrEP), which grants protection to those at risk of exposure to the virus.

The second major concept, HIV care continuum, refers to 5 steps required for an 
HIV-positive person to achieve an undetectable viral load: diagnosis, linkage to care, retention in care, initiation of ART and achieving full viral suppression.

UNAIDS has proposed a $90-90$-90 target in order to reduce transmission and obtain the maximum benefit from antiretroviral therapy. The target calls for $90 \%$ of people living with HIV to be diagnosed, $90 \%$ of those diagnosed to be linked to care and to be commenced on antiretroviral treatment (ART), and $90 \%$ of people on ART to have undetectable viral loads.

But in reality, by the end of 2016, just more than $50 \%$ of HIV infected people globally were on antiretroviral therapy.

In order to understand the gap between reality and the set target, professionals from the UK in 2014, investigated the proportion of people with HIV who were retained at each stage of the cascade in seven high-income countries namely USA, UK, France, Denmark, Netherlands, Australia and Canada (British Columbia). Key points where there was a break in the cascade (a fall-off of $19 \%$ compared to the previous stage) were identified. (Graph 1) Break points indicated areas where national treatment programmes were under-performing.

Data sources included national and UNAIDS surveillance reports, articles in peer-reviewed journals and conference presentations.

Graph 1: Proportion of people with HIV retained at each stage of cascade in 7 highincome countries at the end of 2014

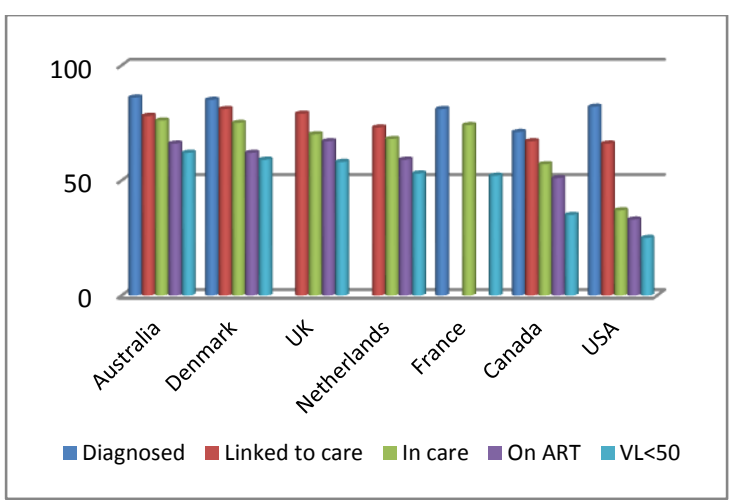

The proportion of HIV infected people who had an undetectable viral load ranged from $62 \%$ in Australia to just $25 \%$ in the United States. In Denmark, the UK, the Netherlands and France, over $50 \%$ of people living with HIV had an undetectable viral load (59\%, 58\%, $53 \%$ and $52 \%$, respectively). Every country, even those who had the best outcomes had attrition at each stage of the treatment cascade.

In the UK and Netherlands this was the high number of undiagnosed infections (21-27\%) not linked to care. In the USA, there was a big gap between the proportion of people linked to care $(66 \%)$ and the percentage retained in care (37\%). (Graph 1)

Graph 2: Cumulative cross-sectional cascade graph of HIV services for PLHIV - 2016

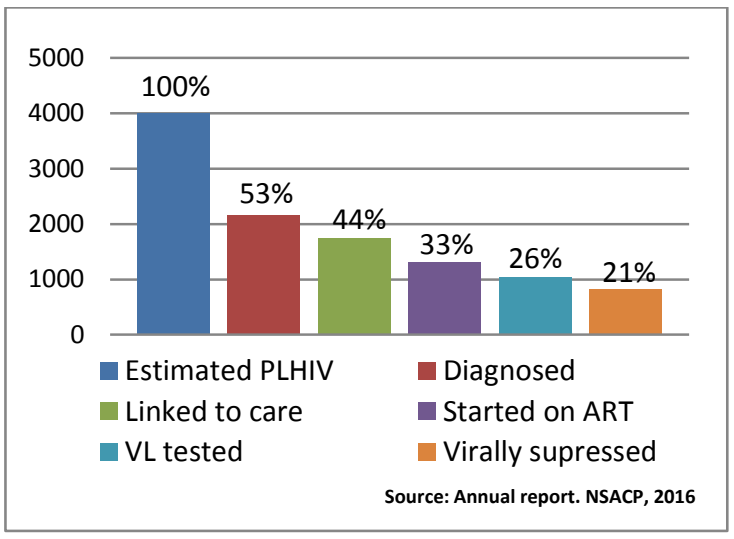

In Sri Lanka, according to the annual report of National STD/AIDS Control Programme in 2016, the biggest gap is seen between the estimated number living with HIV and the number diagnosed. At the same time, more than $78 \%$ of people who had viral load testing, were virally suppressed.

However, even in the best-performing countries viral suppression falls short of the target set by UNAIDS.

\section{Boston patients}

Boston patients were described initially in 2012 and in more detail at the International AIDS Society (IAS) meeting July 2017.

The two men had received donor bone marrow containing haematopoietic stem cells, 
which give rise to all types of blood cells including CD4 T-cells that can harbour HIV. Donors of both men had normal or 'wild-type' stem cells susceptible to HIV infection. The transplants were successful and the blood cells of the two recipients were progressively replaced by donor cells. Both men have maintained viral suppression after the procedure and frequent and extensive testing over the next few years have shown undetectable HIV RNA in plasma, undetectable DNA in peripheral blood mononuclear cells (PBMCs), lymph nodes and gut tissue.

After the men had been apparently HIV-free for 2.6 and 4.3 years respectively, they and their health care providers have agreed to try an ART interruption to see if the virus would return. In 2013, it was reported that the men continued to have undetectable plasma HIV RNA and undetectable integrated HIV DNA in PBMCs after 7 and 15 weeks off ART, raising hopes that the stem cell transplant process might contribute to a functional cure. But these hopes have turned to disappointment as the virus had come back in both men. The first patient experienced HIV re-emergence 12 weeks into his treatment interruption, while the second man showed HIV rebound after he had been off treatment for eight months.

Virus detected after rebound has been genetically similar, suggesting they were clones of only one or a few residual remaining viruses. This implies that even a few remaining HIV infected cells may be enough to allow full viral rebound once protective ART is stopped. One of the biggest challenges to cure HIV is the existence of reservoirs of dormant, HIV-infected cells. Antiretrovirals that were developed in the mid-1990s suppress active virus but these drugs do not affect the HIV reservoirs.

Is it a miracle to cure HIV?

Berlin patient

To date, the only evidence of a medical intervention that has been able to cure HIV infection is the 'Berlin patient' -Timothy Ray
Brown. In 2007 and in 2008, the Seattle-born Brown, living in Berlin, has undergone two bone marrow transplants to treat acute myeloid leukaemia. Because he also had HIV, his German doctor has arranged a stem cell donor who carried two copies of a rare gene mutation CCR5 $\triangle 32$ that confers natural resistance to the virus. The procedure has made him very ill and left him with some permanent nervous system damage. Brown has stopped taking antiretroviral drugs after the first transplant in 2007 and continues to show no signs of HIV.

\section{ICISTEM study}

The results of the ICISTEM, (International Collaboration to guide and investigate the potential for HIV cure by Stem Cell Transplant) a cohort study of 23 patients with HIV and advanced cancers (mainly leukaemia and lymphoma) who have had bone marrow transplants were as follows.

In five of the six patients that were alive, the transplanted HIV-free bone marrow stem cells rapidly replaced the patient's cancerous and HIV-infected cells. Ultrasensitive tests could find no HIV RNA in the blood and no DNA in the reservoirs of these patients. In some patients, HIV DNA undetectability in reservoir cells has happened within a month and in others, HIV DNA levels slowly declined over a period up to a year.

The key to full replacement by new cells appeared to be graft-versus-host disease (GvHD), a condition in which the grafted bone marrow cells 'reject' the body's own cells as foreign- essentially the reverse of what happens in typical transplant rejection. This is normally a condition transplant doctors try to avoid, as it creates severe and in some cases lethal inflammation.

Are the ICISTEM patients cured? The researchers have failed to find a single copy of HIV DNA in one million resting reservoir cells in some of the patients. But their fate is yet to be clear after antiretrovirals are taken off these patients. 
Brown has been off medication for 10 years and the virus has not returned. Before his transplant, he has undergone conditioning, an intense chemotherapy and radiation regimen that destroys the immune system to make room for transplanted immune cells to grow. Could that pre-transplant conditioning have also destroyed his HIV reservoir? Was it the donor whose cells carried rare, CCR5 $\triangle 32$ mutation or was it the GvHD that he developed later?

Until now, attempts to duplicate Brown's cure in other people with HIV who also needed a bone marrow transplant for cancer have not been successful; most of the very ill patients have died either of the cancer or the transplant. But new information kindle hope that Brown's cure can be repeated.

Indeed, at this point, I am reminded of this old Chinese curse: "May you live in interesting times. Well, these are interesting times!! Gazing into the crystal ball at the epidemic's future reveals many exciting achievements.

\section{Other immune therapy modalities}

\section{CAR T cell therapy}

This involves genetically re-engineering the $T$ cells with synthetic receptors called Chimeric Antigen Receptors, or CARs, to kill cancer cells bearing a particular marker. Years ago, scientists have tried T-cell therapies against HIV without much success. Now, building on advances made in cancer and other diseases, there is hope that scientists would revisit these and other approaches that engage the immune system to fight with HIV.

\section{Gene editing}

Successful efforts have been reported in preclinical models to modify blood stem cells using a gene editing technique that employs molecules called zinc-finger nucleases and return the resistant stem cells to repopulate the immune system. This work is part of an effort to use Brown's cure as a blueprint to develop a less-toxic therapy by seeking to genetically engineer resistance in an infected person's own immune cells.

Disabling CCR5 receptor has been of interest of the scientists. Using an animal model of HIV infection the scientists have used a pair of zinc-finger nucleases to disrupt the gene coding for CCR5 in blood stem cells, which are then transplanted back into the animals to repopulate their immune systems with the modified version of CCR5.

CCR5-based approach is focused on mimicking a naturally occurring and safe mutation. Some people of Northern European and Middle Eastern descent have the CCR5 mutation that researchers are hoping to copy through gene editing.

Researchers from Sangamo, the University of Pennsylvania and the Albert Einstein College of Medicine in New York have published results from a clinical trial using one such set of nucleases to disrupt CCR5 in the immune cells of HIV-positive patients. The technique appeared safe and might even be slowing HIV progression, although none of the patients in that trial are truly cured of their infections.

\section{Broadly neutralizing antibodies}

Monoclonal antibodies (mAbs) are currently being explored for HIV therapy and prevention. Approximately, one-third of HIVinfected individuals make HIV neutralizing antibodies and $B$ cells from these individuals were used to isolate first generation HIVneutralizing $\mathrm{mAbs}$.

\section{a) Parenteral Monoclonal Antibody Administration}

One study involved ten patients with chronic HIV infection who had been well suppressed for more than 3 years. All had received infusions of the broadly neutralizing HIVspecific antibody VRCO1 3 days before and 14 and 28 days after the interruption of antiretroviral therapy, and for 7 months thereafter. Ultimately, everybody had rebounded, but the time from discontinuation of antiretroviral therapy to rebound was extended. Median time to plasma viral 
rebound was 39 days in the VRCO1 patients, whereas in historic control subjects, the interval ranged from 11 to 28 days.

\section{b) Topical Monoclonal Antibody Administration}

Topical administration of $m A B$ directly to the genital tract or rectum is another promising approach for HIV prevention. Topical mAbbased microbicides potentially offer several advantages including use of synergistic mAb combinations, ease of application, delivery of concentrated product directly to site where needed, reversibility, few if any side-effects, and cost effectiveness.

Preclinical Studies in Animal Models.

Several studies have demonstrated that topical administration of anti-HIV mAbs can protect macaques from SHIV mucosal challenge. mAbs have been administered vaginally or rectally followed by high dose or repeated low-dose SHIV challenges to the site shortly after mAb delivery. The half-life of mAbs in mucosal secretions after topical delivery of 5-20 mg mAb was about $4 \mathrm{~h}$; animals were protected to varying degrees against the SHIV challenges, and no sideeffects were noted.

\section{Vaccine trials}

It has been decided to go ahead with the prime-boost vaccine trial involving thousands of patients in Africa after showing the twocomponent shot generated a good immune response.

It also follows another large-scale trial already underway in Africa, which uses a modified version of a vaccine that showed a modest 31 percent reduction in infections in Thailand in 2009.

In both cases, researchers anticipate reductions in infection rates of at least 50 percent, and hopefully more.

\section{Check point inhibitors}

These act via a mechanism that cancer cells use to shut down the immune system. Several observational trials are underway of patients with both HIV and melanoma who are receiving such drugs.

In a small cohort of HIV-positive patients with cancers not related to HIV, immunotherapy drugs that block the PD-1 cancer pathway yielded mixed results. But the treatment was safe.

In this prospective 120 day study, twelve patients with undetectable viral loads and cancer (eleven with non-small-cell lung cancer and one with melanoma) received a PD-1 checkpoint blocker after traditional chemotherapy had failed. Three of the 12 patients had a partial response, four were stable, and five experienced disease progression. Although the treatment had little effect on HIV reservoirs in most of the patients, viral loads continued to be undetectable in all patients and one patient has experienced that his reservoir has reduced by $90 \%$.

\section{'Kick and Kill'}

The joint study, known as 'Kick and Kill' involves researchers activating HIV infected cells which are 'asleep', using a cancer drug, which kick-starts the immune system into killing the HIV virus. Bromodomain and extra terminal domain (BET) inhibitors are potent reactivators of latent HIV and could serve as part of a future 'show and kill' clinical strategy. "Multiple clinical trials with various BET inhibitors are currently in early stages specifically for applications in cancer," the researchers note. In addition, treatment with BET inhibitors and low-dose protein kinase $C$ agonists is currently the most effective way to reactivate latent HIV from $\mathrm{CD} 4+\mathrm{T}$ cells isolated from aviremic HIV+ individuals, rendering $B E T$ inhibitors attractive candidates in future clinical trials targeting HIV-1 latency. 
To conclude, I must say that though the crystal ball view is attractive, certainly it is very complex. Until the bright future comes it is still important that we continue to work towards ending HIV transmission and encouraging regular testing as we know that early diagnosis and effective treatment mean people living with HIV can expect long and healthy lives, and transmission rates will be near zero.

We have witnessed the vicious virus. But let's be optimistic that there will be a blossoming day!! Thank you. 